\title{
SEPARATING $p$-BASES AND TRANSCENDENTAL EXTENSION FIELDS
}

\author{
J. N. MORDESON AND B. VINOGRADE
}

\begin{abstract}
Let $L / K$ denote an extension field of characteristic $p \neq 0$. It is known that if $L / K$ has a finite separating transcendence base, then every relative $p$-base of $L / K$ is a separating transcendence base of $L / K$. In this paper we show that when every relative $p$-base of $L / K$ is a separating transcendence base of $L / K$, then the transcendence degree of $L / K$ is finite. We also illustrate the connection between the finiteness of transcendence degree of $L / K$ and the property that $L / K(X)$ is separable algebraic for every relative $p$-base $X$ of $L / K$.
\end{abstract}

Let $L / K$ denote an extension field of characteristic $p>0$. If $X$ is a relative $p$-base such that $L / K(X)$ is separable algebraic, then we call $X$ a separating relative $p$-base. When every relative $p$-base of $L / K$ is a separating relative $p$-base we say that $L / K$ is of type $R_{s}$. Let $S$ denote the set of all intermediate fields of $L / K$. When every element of $S$ is of type $R_{s}$ (with respect to $K$ ), we say that $L / K$ is of type $R_{s}(S)$. This notation extends that used by the authors in [4] where a purely inseparable extension $L / K$ is called type $R$ when $L=K(X)$ for every relative $p$-base $X$, and where it is shown that $L / K$ is of type $R(S)$ if and only if $L / K$ has an exponent.

In this paper we give four theorems that illustrate the connection between type $R_{s}$ and the finiteness of transcendence degree. We make use of relevant results that appear in Mac Lane [3] and Dieudonné [1].

Finitely generated extensions, whose measures of inseparability have recently been analyzed anew by Kraft [2], are a subset of extensions of type $R_{s}(S)$, a fact that follows easily from Theorem 2 below.

LEMMA. $L / K$ is of type $R_{s}$ if and only if there is no intermediate field $L^{\prime}$ of $L / K$ such that $L=L^{\prime}\left(L^{p}\right)$ and $L / L^{\prime}$ is not separable algebraic.

Proof. Suppose $L / K$ is not of type $R_{s}$. Then there exists a relative $p$-base $X$ which is not a separating relative $p$-base. Hence if we set $L^{\prime}=$ $K(X)$, then $L=L^{\prime}\left(L^{p}\right)$ and $L / L^{\prime}$ is not separable algebraic. On the other hand, suppose there exists an intermediate field $L^{\prime}$ of $L / K$ such that

Received by the editors April 5, 1971.

AMS 1970 subject classifications. Primary 12F20; Secondary $12 \mathrm{~F} 99$.

Key words and phrases. Extension fields, separating transcendence bases, relative p-bases.

(c) American Mathematical Society 1972 
$L=L^{\prime}\left(L^{p}\right)$ and $L / L^{\prime}$ is not separable algebraic. Then $L^{\prime}$ contains a relative $p$-base of $L / K$ which is not a separating relative $p$-base. Q.E.D.

Corollary. If $L / K$ is of type $R_{s}$, then $L / L^{\prime}$ is of type $R_{s}$ for every intermediate field $L^{\prime}$ of $L / K$.

Proof. Suppose $L / K$ is of type $R_{s}$ and that $L / L^{\prime}$ is not of type $R_{s}$ for some intermediate field $L^{\prime}$ of $L / K$. Then there exists an intermediate field $L^{\prime \prime}$ of $L / L^{\prime}$ such that $L=L^{\prime \prime}\left(L^{p}\right)$ and $L / L^{\prime \prime}$ is not separable algebraic. This is a contradiction because $L^{\prime \prime}$ is also an intermediate field of $L / K$. Q.E.D.

We call $L / K$ separable when the tensor product $L \otimes_{K} K^{p^{-1}}$ is a field.

THEOREM 1. When $L / K$ is separable, the following statements are equivalent.

(1) $L / K$ is of type $R_{s}(S)$.

(2) $L / K$ is of type $R_{s}$.

(3) $L / K$ has a finite separating transcendence base.

(4) Every relative p-base of $L / K$ is a separating transcendence base of $L / K$.

(5) Every relative p-base of $L / K$ is a transcendence base of $L / K$.

(6) The transcendence degree of $L / K$ equals the imperfection degree of $L / K$, and these are finite.

Proof. $\quad(1) \Rightarrow(2) \Rightarrow(3)$. That (1) implies (2) is immediate. Suppose (2) holds. Then, by [3, Theorem 11, p. 381$], L / K$ has a separating transcendence base $T$. Suppose $T$ is infinite. Let $T_{0}=\left\{t_{1}, t_{2}, \cdots\right\}$ be a denumerable subset of $T$ and set $T^{\prime}=T-T_{0}$ (set difference), $K^{\prime}=K\left(T^{\prime}\right)$. Then $T_{0}$ is a separating transcendence base of $L / K^{\prime} . T_{0}$ is therefore a relative $p$-base of $L / K^{\prime}$, hence the set $T_{0}^{\prime}=\left\{t_{1} t_{2}^{p}, t_{2} t_{3}^{p}, \cdots\right\}$ is a relative $p$-base of $L / K^{\prime}$. By our corollary, $L / K^{\prime}\left(T_{0}^{\prime}\right)$ is separable algebraic. Hence $t_{1} \in K^{\prime}\left(T_{0}^{\prime}, t_{1}^{p}\right)$. However this contradicts the algebraic independence of $T_{0}$ over $K^{\prime}$. Thus $T$ is finite.

$(3) \Rightarrow(4)$. This implication follows from [3, Corollary, p. 385].

(4) $\Rightarrow(5)$. This follows from [3, Theorem 13, p. 383].

$(5) \Rightarrow(6)$. That the transcendence degree of $L / K$ equals the imperfection degree of $L / K$ is immediate. The finiteness condition follows from the equivalence of (4) and (5) and the proof of (2) implies (3).

$(6) \Rightarrow(1)$. By $[3$, Theorem 11, p. 381], we have (3). Hence an application of [3, Theorem 17, p. 386] and [3, Corollary, p. 385] yields (1).

Q.E.D.

When there exists an integer $e \geqq 0$ such that $K\left(L^{p^{\theta}}\right) / K$ is separable but $K\left(L^{p^{o-1}}\right) / K$ is not, then $e$ is called the inseparability exponent of $L / K$, as in [2, p. 111]. When $L / K$ has an inseparability exponent, there exist certain maximal separable intermediate fields of $L / K$ whose construction (for our 
case) is indicated by Dieudonné [1, p. 17] (see also [3, p. 384]) as follows: From a relative $p$-base $X$ of $L / K$ select a subset $Y$ such that $Y^{p^{e}}$ is a relative $p$-base of $K\left(L^{p^{p}}\right) / K$. Since the latter extension is separable, $Y$ is algebraically independent over $K$, and since $K\left(L^{p e}\right) / K\left(Y^{p^{e}}\right)$ is separable, so is $K\left(L^{p^{e}}, Y\right) / K(Y)$. Set $F=K\left(L^{p^{e}}, Y\right)$. Then $F / K$ is a maximal separable intermediate field of $L / K$ and $L / K$ is isomorphic over $F$ to a subfield of the field $F \otimes_{K} K^{p^{-\infty}}$. Such an intermediate field Dieudonné has called distinguished maximal separable. When $L / K$ has a finite relative $p$-base, the degree of $L$ over any distinguished maximal separable intermediate field is Weil's order of inseparability of $L / K([1, \mathrm{pp} .14,17],[2, \mathrm{p} .111])$. The use of the term "distinguished" is consistent with that used by the authors in [5]. This follows from application of [5, Proposition 1.10, p. 5] to the fact that $Y$ is a relative $p$-base of $F / K$ and relatively $p$-independent in $L / K$. In the finitely generated case, a distinguished maximal separable intermediate field $F$ is the same as the optimal separable intermediate field denoted by $K_{0}$ in [2, p. 111]. In fact, $K\left(F^{p^{e}}\right)=K\left(L^{p^{e}}\right)$ holds in our more general context.

For a transcendence base $T$ of $L / K$, let $S_{T}$ denote the maximal separable intermediate field of $L / K(T)$.

THEOREM 2. When $L / K$ is arbitrary, the following statements are equivalent.

(1) $L / K$ is of type $R_{s}(S)$.

(2) $L / K$ has finite transcendence degree and $L / S_{T}$ has an exponent for every transcendence base $T$ of $L / K$.

(3) $L / K$ has finite transcendence degree and $L / S_{T}$ has an exponent for some transcendence base $T$ of $L / K$.

(4) $L / K$ has an inseparability exponent $e$ and $K\left(L^{p^{e}}\right) / K$ has a finite separating transcendence base.

(5) $L / K$ has a distinguished maximal separable intermediate field of type $R_{s}$.

(6) $L / K$ has a distinguished maximal separable intermediate field and every such field is of type $R_{s}$.

Proof. (1) $\Rightarrow(2) \Rightarrow(3)$. If $T$ is any transcendence base of $L / K$, then (1) implies that $L^{\prime} / K$ is of type $R_{s}$ for every intermediate field $L^{\prime}$ of the purely inseparable extension $L / S_{T}$. Since by our corollary $L^{\prime} / S_{T}$ is also of type $R_{s}, L^{\prime} / S_{T}$ is actually of type $R$. Thus $L / S_{T}$ is of type $R(S)$, hence $L / S_{T}$ has an exponent by [4, Corollary, p. 240]. Since $S_{T} / K$ is also of type $R_{s}, T$ is finite by Theorem 1 above. Thus (1) implies (2). That (2) implies (3) is trivial.

(3) $\Rightarrow(1)$. If $L^{\prime}$ is an intermediate field of $L / K$, then a transcendence base $Z^{\prime}$ of $L^{\prime} / K$ can be extended to a transcendence base $Z$ of $L / K$. Let $T$ 
be the transcendence base of $L / K$ satisfying (3). Since $T$ is finite, there exists a positive integer $m$ such that $T^{p^{m}} \subseteq S_{Z}$. Hence $L / S_{Z}$ has an exponent. Now $S_{Z} \supseteq S_{Z^{\prime}}, S_{Z} / K\left(Z^{\prime}\right)$ is separable and $S_{Z^{\prime}} / K\left(Z^{\prime}\right)$ is, in particular, relatively perfect. Hence $S_{Z} / S_{Z}$ is separable by [1, Proposition 6, p. 8]. Thus $L^{\prime} \cap S_{Z}=S_{Z^{\prime}}$. Since $L / S_{Z}$ has an exponent, say $n, L^{\prime p^{n}} \subseteq L^{\prime} \cap S_{Z}=$ $S_{Z^{\prime}}$. Thus property (3) is inherited by every intermediate field of $L / K$. Hence it suffices to show that (3) implies $L / K$ is of type $R_{s}$. Now $T$ is finite, so $S_{T} / K$ is of type $R_{s}(S)$ by Theorem 1 . Since $L^{p^{e}} \subseteq S_{T}$ for some integer $e \geqq 0$, we have that $K\left(L^{p^{c}}\right) / K$ is of type $R_{s}$. If $X$ is any relative $p$ base of $L / K, X^{p^{e}}$ contains a relative $p$-base of $K\left(L^{p^{e}}\right) / K$. Hence $K\left(L^{p^{e}}\right) / K\left(X^{p^{e}}\right)$ is separable algebraic, whence $K\left(L^{p^{e}}, X\right) / K(X)$ is separable algebraic. Since $L=K\left(L^{p^{e}}, X\right)$ and $X$ was arbitrary, we have that $L / K$ is of type $R_{s}$.

(3) $\Leftrightarrow(4)$. That (4) implies (3) follows easily. To show that (3) implies (4), note that by (3), $L / K$ has an inseparability exponent, say $e$. Since (3) and (1) are equivalent, $K\left(L^{p^{e}}\right) / K$ is of type $R_{s}$. Hence, by Theorem 1 , $K\left(L^{p^{e}}\right) / K$ has a finite separating transcendence base.

(4) $\Leftrightarrow(5)$. To show (5) implies (4), let $F=K\left(L^{p}, Y\right)$ be a distinguished maximal separable intermediate field such that $F / K$ is of type $R_{s}$. Since $F / K$ is separable, every relative $p$-base of $F / K$ is a finite separating transcendence base. Hence $Y$ is a finite separating transcendence base of $F / K$. Thus $Y^{p^{e}}$ is a finite separating transcendence base of $K\left(L^{p^{e}}\right) / K$. To show that (4) implies (5), note that $L / K\left(L^{p^{e}}\right)$ has an exponent and $K\left(L^{p^{e}}\right) / K$ has a finite separating transcendence base. By (3) implies (1), $L / K$ is of type $R_{s}(S)$. Hence $F / K$ is of type $R_{s}$.

$(4) \Leftrightarrow(6)$. (6) implies (5) trivially and we have proved (5) implies (4). Hence (6) implies (4). Assume (4). Now, all distinguished maximal separable intermediate fields contain $K\left(L^{p^{e}}\right) / K$. Hence the proof that (4) implies (5) applies to every such distinguished intermediate field. Q.E.D.

THEOREM 3. When $L / K$ has finite transcendence degree, the following statements are equivalent.

(1) $L / K$ is of type $R_{s}$.

(2) $L / S_{T}$ is of type $R$ for every transcendence base $T$ of $L / K$.

(3) $L / S_{T}$ is of type $R$ for some transcendence base $T$ of $L / K$.

Proof. That (1) implies (2) follows from the Corollary. That (2) implies (3) is trivial. To show that (3) implies (1), let $X$ be a relative $p$-base of $L / K$. Then $X$ contains a relative $p$-base of $L / S_{T}$. Since $L / S_{T}$ is of type $R, L=S_{T}(X) \supseteq K(T, X)$. Thus $L / K(T, X)$ is separable algebraic and by hypothesis $T$ is finite. If $L / K(X)$ is not separable algebraic, then $K(T, X) / K(X)$ is not separable algebraic. Then $K(T, X) / K(X)$ has a nonempty relative $p$-base, because $K(T, X) / K(X)$ is finitely generated. But 
since $L / K(T, X)$ is separable algebraic, $L / K(X)$ has a nonempty relative $p$-base, contrary to the fact that $L / K(X)$ is relatively perfect. Q.E.D.

When an extension field has a separating transcendence base, we say it is separably generated.

THEOREM 4. When $L / K$ contains a separable intermediate field $F / K$ such that $L / F$ is finite degree purely inseparable, the following statements are equivalent.

(1) $L / K$ is of type $R_{s}(S)$.

(2) $L / K$ is of type $R_{s}$.

(3) The transcendence degree of $L / K$ is finite and $F / K$ is separably generated.

(4) The imperfection degree of $L / K$ is finite and $F / K$ is separably generated.

(5) $F / K$ is of type $R_{s}$.

(6) $K\left(L^{p^{e}}\right) / K$ is of type $R_{s}$ for some integer $e \geqq 0$.

Proof. $\quad(1) \Rightarrow(2) \Rightarrow(3)$. That (1) implies (2) is immediate. Suppose (2) holds. Let $X$ be a relative $p$-base of $L / F$. Then $X^{p^{e}} \subseteq F$ for some integer $e \geqq 0$ and $X$ is finite. Let $Y$ be a relative $p$-base of $F / K$. Since $L / K$ is of type $R_{s}$ and $X \cup Y$ contains a relative $p$-base of $L / K, L / K(X, Y)$ is separable algebraic. Hence $K\left(L^{p^{e}}, Y\right) / K\left(X^{p^{e}}, Y\right)$ is separable algebraic. Now $K\left(L^{p^{e}}, Y\right) \subseteq F=K\left(F^{p^{e}}, Y\right) \subseteq K\left(L^{p^{e}}, Y\right)$. Thus $F=K\left(L^{p^{e}}, Y\right)$, so $F / K\left(X^{p^{e}}, Y\right)$ is separable algebraic. Since $F / K$ is separable, $F / K(Y)$ is separable. Hence $K\left(Y, X^{p^{e}}\right) / K(Y)$ is separable and finitely generated. If the latter extension has a nonempty relative $p$-base, then we contradict the fact that $F / K(Y)$ is relatively perfect and $F / K\left(Y, X^{p^{e}}\right)$ is separable algebraic. Hence $F / K(Y)$ is separable algebraic, so $F / K$ is of type $R_{s}$. Since $F / K$ is also separable, it has a finite separating transcendence base by Theorem 1 . Since $L / F$ is algebraic, $L / K$ has a finite transcendence base.

$(3) \Rightarrow(4) \Rightarrow(5)$. That (3) implies (4) is routine. Suppose (4) holds. Then the transcendence degree of $F / K$ must be finite. Hence by Theorem 1 , $F / K$ is of type $R_{s}$.

$(5) \Rightarrow(6)$. Let $e$ be a positive integer such that $L^{p^{e}} \subseteq F$. Since $F / K$ is of type $R_{s}, F / K$ is of type $R_{s}(S)$. Hence $K\left(L^{p^{e}}\right) / K$ is of type $R_{s}$.

(6) $\Rightarrow(1)$. Since $K\left(L^{p^{e}}\right) / K$ is of type $R_{s}, K\left(L^{p^{e}}\right) / K\left(X^{p^{e}}\right)$ is separable algebraic for every relative $p$-base $X$ of $L / K$. Hence $K\left(L^{p^{e}}, X\right) / K(X)$ is separable algebraic, that is $L / K$ is of type $R_{s}$. Hence, as in the proof of (2) implies (3), $L / K$ has finite transcendence degree and $F / K$ is of type $R_{s}$. Thus, replacing $S_{T}$ by $F$ in Theorem 2, we have that (1) holds. Q.E.D.

\section{REFERENCES}

1. J. Dieudonné, Sur les extensions transcendantes séparables, Summa Brasil. Math. 2 (1947), no. 1, 1-20. MR 10, 5. 
2. H. Kraft, Inseparable Körpererweiterungen, Comment. Math. Helv. 45 (1970), 110-118. MR 41 \#5333.

3. S. Mac Lane, Modular fields. I: Separating transcendence bases, Duke Math. J. 5 (1939), 372-393.

4. J. Mordeson and B. Vinograde, Exponents and intermediate fields of purely inseparable extensions. J. Algebra 17 (1971), 238-243.

5. - Structure of arbitrary purely inseparable extension fields, Lecture Notes in Math., vol. 173, Springer-Verlag, New York, 1970.

Department of Mathematics, Creighton University, Omaha, Nebraska 68131

Department of Mathematics, Iowa State University, Ames, Iowa 50010 